\title{
Comment on "Principal Role of the Stepwise Aggregation Mechanism in Ionic Surfactant Solutions Near the Critical Micelle Concentration. Molecular Dynamics Study"
}

\author{
Ana Vila Verde ${ }^{1}$ \\ ${ }^{1}$ FOM institute AMOLF, Science Park 102, 1098 XG Amsterdam, The Netherlands; University of Minho, Department of \\ Physics, Campus de Gualtar 4710 - 057 Braga, Portugal, a.vilaverde@amolf.nl
}

November 2010

In a recent paper, ${ }^{1}$ Burov et al. report on the mechanisms by which micelles of ionic surfactants change size near the critical micellar concentration. From unbiased molecular dynamics simulations of model surfactants they conclude that monomer fission and fusion are the predominant mechanisms of micelle size change, for two reasons: (1) monomer fission and fusion represent $97 \%$ of all fission and fusion events and (2) solving a system of kinetic equations that neglects fission and fusion of larger fragments recovers the distribution of micelle sizes observed directly from simulation. While argument (1) unequivocally supports the main conclusion of the paper, here we will show that argument (2) is flawed and may not be used to assess the predominance of any given mechanism.

Briefly, the system of kinetic equations in question reflects the fact that the simulations were performed at constant total number of particles and were long enough to reach equilibrium. The kinetic equations thus require that both the total surfactant concentration and the concentration of micelles of any size remain constant. This system of equations contains only two types of variables: the concentration of each micellar species and the rate constants for fission and fusion of micelles of any given size. Using the rate constants for monomer fission and fusion obtained from the simulation, they solve that system of equations for the micelle size distribution (the concentration $C_{n}$ of micelles with $n$ molecules). They find that $C_{n}$ obtained using this kinetic model is almost identical to that determined directly from simulation, and take this as evidence that fission and fusion of large fragments are not significant processes of micelle size change.

A thermodynamic argument shows that this conclusion may not be made. The relative concentration of different micellar species in equilibrium systems depends exclusively on their relative free energy; it does not depend on the path by which one micellar species con- verts into another. In other words, equilibrium systems obey detailed balance: the forward and backward reaction rates of any processes connecting any two states are identical. As an example, let us take a system that may exist in two states (A and B) connected by two processes (1 and 2) as illustrated in figure 1. Detailed balance tells us that $C_{A} k_{f, 1}=C_{B} k_{b, 1}$. If the two rate constants are known, clearly we can recover $C_{B}$ for any given $C_{A}$. As detailed balance is always obeyed in equilibrium, $C_{A} k_{f, 2}=C_{B} k_{b, 2}$ is also true and will lead to the same $C_{B}$ for the same initial $C_{A}$, even if $k_{f, 1} \gg k_{f, 2}$ and $k_{b, 1} \gg k_{b, 2}$ (if process 1 is much more frequent than 2 ). It

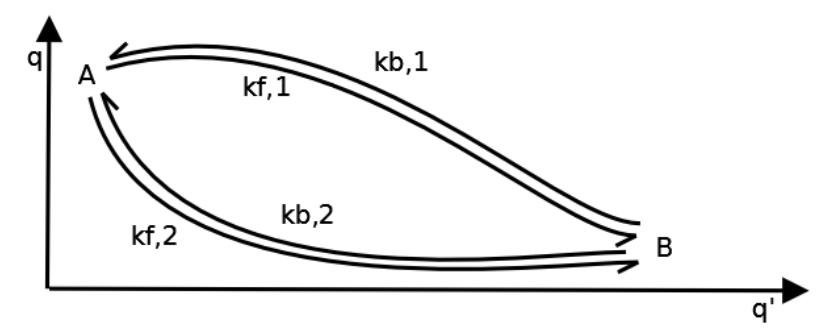

Figure 1: Diagram of a system described by two reaction coordinates $q$ and $q^{\prime}$ with two stable states $A$ and $B$ connected by two distinct processes 1 and $2 . k_{f, 1}$ and $k_{f, 2}$ are the forward rate constants for either process; $k_{b, 1}$ and $k_{b, 2}$ the backward rates.

follows that the solution of a system of kinetic equations that includes only monomer fission and fusion will always recover the correct $C_{n}$ distribution even when other processes of micelle size change are abundant, provided that the rate constants for monomer fission and fusion of micelles of any size present in the simulation are non-zero. In the thought experiment described in ref. 1 where additional transitions are switched on at some time $t_{o n}$, the authors argue that the distribution $C_{n}$ would relax to a new form at $t>t_{\text {on }}$. However, such a relaxation would 
violate detailed balance and is therefore impossible in this equilibrium system.

In summary, the relative importance of different processes of micelle size change may be assessed by calculating the fraction of particular fission and fusion events (e.g. monomer addition and removal) relative to the total number of events in the system, as Burov et al. correctly did. Comparisons between $C_{n}$ distributions directly obtained from simulation and as the solution of kinetic models are not appropriate to make this assessment.

\section{Acknowledgments}

This work is part of the research program of FOM and is made possible by financial support from the Netherlands Organization for Scientific Research (NWO). AV acknowledges support from Senter-Novem and the Dutch Ministry of Economic Affairs through grant DFN0642300 for a joint FOM-Unilever project.

\section{References}

[1] Burov, S. V.; Vanin, A. A.; Brodskaya, E. N. J. Phys. Chem. B 2009, 113, 10715. 